\title{
Existence results for a supercritical Neumann problem with a convex-concave non-linearity
}

\author{
Abbas Moameni ${ }^{1} \cdot$ Leila Salimi $^{2}$ \\ Received: 12 July 2018 / Accepted: 24 November 2018 / Published online: 4 December 2018 \\ () Fondazione Annali di Matematica Pura ed Applicata and Springer-Verlag GmbH Germany, part of Springer \\ Nature 2018
}

\section{Abstract}

We shall consider the following semi-linear problem with a Neumann boundary condition

$$
-\Delta u+u=a(|x|)|u|^{p-2} u-b(|x|)|u|^{q-2} u, \quad x \in B_{1},
$$

where $B_{1}$ is the unit ball in $\mathbb{R}^{N}, N \geq 2, a, b$ are nonnegative radial functions, and $p, q$ are distinct numbers greater than or equal to 2 . We shall assume no growth condition on $p$ and $q$. Our plan is to use a new variational principle that allows one to deal with problems with supercritical Sobolev non-linearities. Indeed, we first find a critical point of the EulerLagrange functional associated with this equation over a suitable closed and convex set. Then we shall use this new variational principle to deduce that the restricted critical point of the Euler-Lagrange functional is an actual critical point.

Keywords Semi-linear elliptic problems · Calculus of variations · Variational principles

\section{Mathematics Subject Classification 35J15 · 58E30}

\section{Introduction}

In this paper, we consider the Neumann problem

$$
\begin{cases}-\Delta u+u=a(|x|)|u|^{p-2} u-b(|x|)|u|^{q-2} u, & x \in B_{1} \\ u>0, & x \in B_{1}, \\ \frac{\partial u}{\partial v}=0, & x \in \partial B_{1},\end{cases}
$$

where $B_{1}$ is the unit ball centered at the origin in $\mathbb{R}^{N}, N \geq 2$ and $p, q$ are distinct numbers greater than or equal to 2 . The functions $a$ and $b$ are assumed to be nonnegative and radially

Abbas Moameni is pleased to acknowledge the support of the National Sciences and Engineering Research Council of Canada. Leila Salimi is supported by a Grant from IPM (No. 96470045).

$\bowtie$ Abbas Moameni

momeni@math.carleton.ca

1 School of Mathematics and Statistics, Carleton University, Ottawa, ON K1S 5B6, Canada

2 Schoo of Mathematics Institute for Research in Fundamental Sciences (IPM), P.O. Box 19395-5746, Tehran, Iran 
monotone. We shall assume no growth conditions on $p$ and $q$, and therefore, this problem can be supercritical.

Our plan is to prove the existence of at least one positive radially non-decreasing solution for problem (1). We would like to remark that there have been several studies on the existence of positive radially non-decreasing solutions for problem (1) when the function $b$ is identically zero, i.e.,

$$
\begin{cases}-\Delta u+u=a(|x|)|u|^{p-2} u, & x \in B_{1} \\ u>0, & x \in B_{1}, \\ \frac{\partial u}{\partial v}=0, & x \in \partial B_{1},\end{cases}
$$

In most of these studies, when $p$ is supercritical, the authors took advantage of the fact that the function on the right-hand side of equation (2) is an increasingly monotone function in terms of $u$. However, this property is lost in (1) as the right-hand side of this equation is the difference of two monotone functions in terms of $u$. Here is one of the main results in this paper.

Theorem 1.1 Let $p$ and $q$ be two distinct real numbers with $p>q \geq 2$. Assume that functions $a$ and $b$ satisfy the following conditions:

H1. $a \in L^{1}(0,1)$ is non-decreasing and $a(r)>0$ for a.e. $r \in[0,1]$.

H2. $b \in L^{1}(0,1)$ is nonnegative and non-increasing on $[0,1]$.

Then problem (1) admits at least one radially non-decreasing positive solution.

We shall show that a similar result as in Theorem 1.1 also holds for $p<q$ (see Theorem 3.7). Here is an example satisfying the assumptions of Theorem 1.1:

$$
\begin{cases}-\Delta u+u=|x|^{\alpha}|u|^{p-2} u-\frac{\mu}{|x|^{\beta}}|u|^{q-2} u, & x \in B_{1} \\ u>0, & x \in B_{1}, \\ \frac{\partial u}{\partial v}=0, & x \in \partial B_{1} .\end{cases}
$$

where $\alpha, \mu \geq 0$ and $0 \leq \beta<N$.

To prove Theorem 1.1, we utilize an abstract variational principle from [26] (see also [27]). To be more specific, let $V$ be a reflexive Banach space, $V^{*}$ its topological dual and let $K$ be a non-empty convex and weakly closed subset of $V$. Assume that $\Psi: V \rightarrow \mathbb{R} \cup\{+\infty\}$ is a proper, convex, lower semi-continuous function which is Gâteaux differentiable on K. The Gâteaux derivative of $\Psi$ at each point $u \in K$ will be denoted by $D \Psi(u)$. The restriction of $\Psi$ to $K$ is denoted by $\Psi_{K}$ and defined by

$$
\Psi_{K}(u)= \begin{cases}\Psi(u), & u \in K, \\ +\infty, & u \notin K .\end{cases}
$$

For a given functional $\Phi \in C^{1}(V, \mathbb{R})$ denote by $D \Phi \in V^{*}$ its derivative and consider the functional $I_{K}: V \rightarrow(-\infty,+\infty]$ defined by

$$
I_{K}(u):=\Psi_{K}(u)-\Phi(u) .
$$

According to Szulkin [30], we have the following definition for critical points of $I_{K}$.

Definition 1.2 A point $u_{0} \in V$ is said to be a critical point of $I_{K}$ if $I_{K}\left(u_{0}\right) \in \mathbb{R}$ and if it satisfies the following inequality

$$
\left\langle D \Phi\left(u_{0}\right), u_{0}-v\right\rangle+\Psi_{K}(v)-\Psi_{K}\left(u_{0}\right) \geq 0, \quad \forall v \in V,
$$

where $\langle.,$.$\rangle is the duality pairing between V$ and its dual $V^{*}$. 
We also recall the notion of the point-wise invariance condition from [26].

Definition 1.3 We say that the triple $(\Psi, K, \Phi)$ satisfies the point-wise invariance condition at a point $u_{0} \in V$ if there exists a convex Gâteaux differentiable function $G: V \rightarrow \mathbb{R}$ and a point $v_{0} \in K$ such that

$$
D \Psi\left(v_{0}\right)+D G\left(v_{0}\right)=D \Phi\left(u_{0}\right)+D G\left(u_{0}\right) .
$$

We shall now recall the following variational principle recently established in [26] (see also [27]).

Theorem 1.4 Let $V$ be a reflexive Banach space and $K$ be a convex and weakly closed subset of $V$. Let $\Psi: V \rightarrow \mathbb{R} \cup\{+\infty\}$ be a convex, lower semi-continuous function which is Gâteaux differentiable on $K$ and let $\Phi \in C^{1}(V, \mathbb{R})$. Assume that the following two assertions hold:

(i) The functional $I_{K}: V \rightarrow \mathbb{R} \cup\{+\infty\}$ defined by $I_{K}(u):=\Psi_{K}(u)-\Phi(u)$ has a critical point $u_{0} \in V$ as in Definition 1.2, and;

(ii) the triple $(\Psi, K, \Phi)$ satisfies the point-wise invariance condition at the point $u_{0}$.

Then $u_{0} \in K$ is a solution of the equation

$$
D \Psi(u)=D \Phi(u) .
$$

We would like to remark that a particular version of Theorem 1.4 has been successfully applied to the well-known Ambrosetti-Brezis-Cerami concave-convex problem [3] to obtain multiplicity results for supercritical non-linearities (see [24] for more details).

For the convenience of the reader, by choosing appropriate functions $\Psi, \Phi$ and a convex set $K$ corresponding to our problem (1), we shall provide a proof to a particular case of Theorem 1.4 applicable to this problem.

We now recall some prior works related to (2). In [6], the authors considered the following problem

$$
\begin{cases}-\Delta u+u=|u|^{p}, & x \in B_{1} \\ u>0, & x \in B_{1}, \\ \frac{\partial u}{\partial v}=0, & x \in \partial B_{1},\end{cases}
$$

and proved the existence of multiple radial solutions as $p \rightarrow+\infty$. They also showed the existence of radial solutions to (6) whose Laplacians weakly converge to measures concentrating at interior spheres, with a simple reflection rule. Also in [28], the authors considered a variant of (2) where $|u|^{p}$ is replaced with $a(|x|) f(u)$ where $a$ and $f$ are positive and non-decreasing functions. They proved the existence of a positive and radially non-decreasing solution for this problem when there is no growth condition on $f$. In [7], by using the topological and variational arguments, the authors treated a more general problem without imposing any growth condition on $f$ and proved the existence of positive non-decreasing radial solutions. They have also addressed the existence of a non-constant solution in case the problem admits trivial constant solutions. These results were extended to the $p$-Laplace version in $[13,29]$. In recent papers $[10,11]$, the authors studied the $p$-laplace version when the right-hand side of (6) is replaced with more general non-linearities and the domain is either a ball or an annulus. In particular, a conjecture about the existence of solutions with certain qualitative properties (raised in [7]) was answered in [10]. The methods of [23] were extended to prove results regarding multilayer radials solutions in [6]. The next work related to (6) is [8] where they considered

$$
\begin{cases}-\Delta u+u=g(|x|)|u|^{p-1}, & x \in \Omega \\ u>0, & x \in \Omega, \\ \frac{\partial u}{\partial v}=0, & x \in \partial \Omega,\end{cases}
$$


where $\Omega$ is an annulus in $\mathbb{R}^{N}$ :

$$
\Omega:=\left\{x \in \mathbb{R}^{N}: a<|x|<b\right\},
$$

and $g \in L^{1}(a, b), g(r)>0$ a.e. in $(a, b)$ and $p>2$. They proved that for every $p$ large enough, problem (7) admits at least three distinct redial solutions. Also in [9] the elliptic system of the form

$$
\begin{cases}-\Delta u+u=a(|x|) f(u, v), & x \in B_{1} \\ -\Delta v+v=\tilde{a}(|x|) g(u, v), & x \in B_{1} \\ \frac{\partial u}{\partial v}=\frac{\partial v}{\partial v}=0, & x \in \partial B_{1},\end{cases}
$$

has been considered, where $a, \tilde{a}$ are assumed to be radially, nonnegative and non-decreasing weights and $f, g$ are non-decreasing in each component. With some assumptions on $f$ and $g$, they proved the existence of at least one couple of non-decreasing non-trivial solutions for (8).

Neumann boundary problems with subcritical non-linearities by means of Sobolev spaces have been studied in several papers, among which we refer to $[1,2,4,20,21]$ and the references therein.

Note that as the right-hand side of equation (2) is an non-decreasing function of $u$, one can write it as a gradient of a convex function, namely $\varphi$. Now if $\varphi^{*}$ is the Fenchel dual of $\varphi$, then by considering the critical points of the functional

$$
I(u)=\varphi^{*}(A u)-\varphi(u),
$$

where $A u=-\Delta u+u$, one can prove the existence of a solution for the equation (See $[14,15,25,26])$. We shall refer to [14] in particular where this new functional $I$ was used to study problem (2) for non-radial domains.

The paper is organized as follows. In the next section, we shall recall some preliminaries from convex analysis and non-smooth critical point theory. Section 3 is devoted to the proof of our main results. In the last section, we shall also discuss the case of non-constant solutions when the functions $a$ and $b$ are constants.

\section{Preliminaries}

In this section, we recall some important definitions and results from convex analysis and minimax principles for lower semi-continuous functions.

Let $V$ be a real Banach space and $V^{*}$ its topological dual and let $\langle.$, . $\rangle$ be the pairing between $V$ and $V^{*}$. The weak topology on $V$ induced by $\langle.,$.$\rangle is denoted by \sigma\left(V, V^{*}\right)$. A function $\Psi: V \rightarrow \mathbb{R}$ is said to be weakly lower semi-continuous if

$$
\Psi(u) \leq \liminf _{n \rightarrow \infty} \Psi\left(u_{n}\right),
$$

for each $u \in V$ and any sequence $u_{n}$ approaching $u$ in the weak topology $\sigma\left(V, V^{*}\right)$. Let $\Psi: V \rightarrow \mathbb{R} \cup\{+\infty\}$ be a proper convex function. The subdifferential $\partial \Psi$ of $\Psi$ is defined to be the following set-valued operator: If $u \in \operatorname{Dom}(\Psi)=\{v \in V ; \Psi(v)<+\infty\}$, set

$$
\partial \Psi(u)=\left\{u^{*} \in V^{*} ;\left\langle u^{*}, v-u\right\rangle+\Psi(u) \leq \Psi(v) \text { for all } v \in V\right\}
$$

and if $u \notin \operatorname{Dom}(\Psi)$, set $\partial \Psi(u)=\varnothing$. If $\Psi$ is Gâteaux differentiable at $u$, denote by $D \Psi(u)$ the derivative of $\Psi$ at $u$. In this case, $\partial \Psi(u)=\{D \Psi(u)\}$. 
We shall now recall some notations and results for the minimax principles for lower semi-continuous functions.

Definition 2.1 Let $V$ be a real Banach space, $\Phi \in C^{1}(V, \mathbb{R})$ and $\Psi: V \rightarrow(-\infty,+\infty]$ be proper (i.e., $\operatorname{Dom}(\Psi) \neq \emptyset$ ), convex and lower semi-continuous. A point $u \in V$ is said to be a critical point of

$$
I:=\Psi-\Phi
$$

if $u \in \operatorname{Dom}(\Psi)$ and if it satisfies the inequality

$$
\langle D \Phi(u), u-v\rangle+\Psi(v)-\Psi(u) \geq 0, \quad \forall v \in V .
$$

Definition 2.2 We say that $I$ satisfies the Palais-Smale compactness condition (PS) if every sequence $\left\{u_{n}\right\}$ such that

- $I\left[u_{n}\right] \rightarrow c \in \mathbb{R}$,

- $\left\langle D \Phi\left(u_{n}\right), u_{n}-v\right\rangle+\Psi(v)-\Psi\left(u_{n}\right) \geq-\epsilon_{n}\left\|v-u_{n}\right\|, \quad \forall v \in V$.

where $\epsilon_{n} \rightarrow 0$, then $\left\{u_{n}\right\}$ possesses a convergent subsequence.

The following two theorems are due to Szulkin [30].

Theorem 2.3 (Mountain pass theorem) Suppose that $I: V \rightarrow(-\infty,+\infty]$ is of the form (9) and satisfies the Palais-Smale condition and the mountain pass geometry (MPG):

1. $I(0)=0$.

2. there exists $e \in V$ such that $I(e) \leq 0$.

3. there exists some $\rho$ such that $0<\rho<\|e\|$ and for every $u \in V$ with $\|u\|=\rho$ one has $I(u)>0$.

Then I has a critical value $c$ which is characterized by

$$
c=\inf _{g \in \Gamma} \sup _{t \in[0,1]} I[g(t)],
$$

where $\Gamma=\{g \in C([0,1], V): g(0)=0, g(1)=e\}$.

Theorem 2.4 Suppose that $I: V \rightarrow(-\infty,+\infty]$ is of the form (9) and satisfies the PalaisSmale condition. If I is bounded from below, then $c=\inf _{u \in V} I(u)$ is a critical value.

\section{Existence results}

In this section, we first prove an adapted version of Theorem 1.4 applicable specifically to our problem, and then, we proceed with the proof of our main results.

Throughout this section, we always assume that conditions $H 1$ and $H 2$ in Theorem 1.1 hold, and the real numbers $p, q$ are greater than or equal to 2 . Let $H_{\text {rad }}^{1}\left(B_{1}\right)$ be the space of radial function in the Sobolev space $H^{1}\left(B_{1}\right)$. Consider the Banach space $V=H_{\mathrm{rad}}^{1}\left(B_{1}\right) \cap L_{b}^{q}\left(B_{1}\right) \cap$ $L_{a}^{p}\left(B_{1}\right)$ with

$$
L_{a}^{p}\left(B_{1}\right):=\left\{u: \int a(|x|)|u|^{p} \mathrm{~d} x<\infty\right\}, \quad \& \quad L_{b}^{p}\left(B_{1}\right):=\left\{u: \int b(|x|)|u|^{p} \mathrm{~d} x<\infty\right\},
$$

equipped with the following norm

$$
\|u\|_{V}=\|u\|_{H^{1}}+\|u\|_{L_{a}^{p}}+\|u\|_{L_{b}^{q}},
$$


where

$$
\|u\|_{L_{a}^{p}}=\left(\int_{B_{1}} a(|x|)|u|^{p} \mathrm{~d} x\right)^{\frac{1}{p}}, \quad \& \quad\|u\|_{L_{b}^{q}}=\left(\int_{B_{1}} b(|x|)|u|^{q} \mathrm{~d} x\right)^{\frac{1}{q}} .
$$

Note that the duality pairing between $V$ and its dual $V^{*}$ is defined by

$$
\left\langle u, u^{*}\right\rangle=\int_{\Omega} u(x) u^{*}(x) \mathrm{d} x, \quad \forall u \in V, \forall u^{*} \in V^{*} .
$$

Our plan is to apply Theorem 1.4 to the Euler-Lagrange functional corresponding to problem (1), i.e.,

$$
E(u):=\frac{1}{2} \int_{B_{1}}\left(|\nabla u|^{2}+u^{2}\right) \mathrm{d} x+\frac{1}{q} \int_{B_{1}} b(x) u^{q} \mathrm{~d} x-\frac{1}{p} \int_{B_{1}} a(|x|)|u|^{p} \mathrm{~d} x,
$$

over the set

$$
K=\{u \in V: u \geq 0, u \text { is non-decreasing with respect to the radius } r=|x|\} .
$$

To adapt Theorem 1.4 to our case, define $\Psi: V \rightarrow \mathbb{R}$ and $\Phi: V \rightarrow \mathbb{R}$ by

$$
\Psi(u)=\frac{1}{2} \int_{B_{1}}\left(|\nabla u|^{2}+u^{2}\right) \mathrm{d} x+\frac{1}{q} \int_{B_{1}} b(|x|) u^{q} \mathrm{~d} x,
$$

and

$$
\Phi(u)=\frac{1}{p} \int_{B_{1}} a(|x|)|u|^{p} \mathrm{~d} x .
$$

We remark that even though $\Phi$ is not even well defined on $H^{1}\left(B_{1}\right)$, but it is continuously differentiable on the space $V=H_{\text {rad }}^{1}\left(B_{1}\right) \cap L_{b}^{q}\left(B_{1}\right) \cap L_{a}^{p}\left(B_{1}\right)$. Finally, let us introduce the functional $E_{K}(u): V \rightarrow(-\infty,+\infty]$ defined by

$$
E_{K}(u):=\Psi_{K}(u)-\Phi(u)
$$

where

$$
\Psi_{K}(u)= \begin{cases}\Psi(u), & u \in K \\ +\infty, & u \notin K\end{cases}
$$

Note that $E_{K}$ is indeed the Euler-Lagrange functional corresponding to (1) restricted to $K$. Here is an adapted version of Theorem 1.4 applicable to our case.

Theorem 3.1 Let $V=H_{\mathrm{rad}}^{1}\left(B_{1}\right) \cap L_{b}^{q}\left(B_{1}\right) \cap L_{a}^{p}\left(B_{1}\right)$ and let $K$ be the convex and closed subset of $V$ given in (11). Suppose the following two assertions hold:

(i) The functional $E_{K}: V \rightarrow \mathbb{R} \cup\{+\infty\}$ defined in (12) has a critical point $\bar{u} \in V$ as in Definition 2.1, and;

(ii) there exists $\bar{v} \in K$ with $\frac{\partial \bar{v}}{\partial v}=0$ on the boundary of $B_{1}$ such that

$$
-\Delta \bar{v}+\bar{v}+b(x)|\bar{v}|^{q-2} \bar{v}=D \Phi(\bar{u})=a(|x|)|\bar{u}|^{p-2} \bar{u},
$$

in the weak sense, i.e.,

$$
\int_{\Omega} \nabla \bar{v} \cdot \nabla \eta \mathrm{d} x+\int_{\Omega}\left(\bar{v}+b(x)|\bar{v}|^{q-2} \bar{v}\right) \eta \mathrm{d} x=\int_{\Omega} a(|x|)|\bar{u}|^{p-2} \bar{u} \eta \mathrm{d} x, \quad \forall \eta \in V .
$$


Then $\bar{u} \in K$ is a weak solution of the equation

$$
-\Delta u+u=a(|x|)|u|^{p-2} u-b(x)|u|^{q-2} u,
$$

with the Neumann boundary condition.

Proof Since $\bar{u}$ is a critical point of $E_{K}$, it follows from Definition 2.1 that

$$
\langle D \Phi(\bar{u}), \bar{u}-v\rangle+\Psi_{K}(v)-\Psi_{K}(\bar{u}) \geq 0, \quad \forall v \in V .
$$

On the other hand, by (ii), there exists $\bar{v} \in K$ satisfying

$$
\left\{\begin{array}{lr}
-\Delta \bar{v}+\bar{v}+b(x)|\bar{v}|^{q-2} \bar{v}=a(|x|)|\bar{u}|^{p-2} \bar{u}, & x \in B_{1} \\
\frac{\partial \bar{v}}{\partial v}=0, & x \in \partial B_{1},
\end{array}\right.
$$

in the weak sense. By setting $v=\bar{v}$ in (15), we obtain that

$$
\begin{aligned}
& \frac{1}{2} \int_{B_{1}}\left(|\nabla \bar{v}|^{2}+\bar{v}^{2}\right) \mathrm{d} x+\frac{1}{q} \int_{B_{1}} b(x)|\bar{v}|^{q} \mathrm{~d} x-\frac{1}{2} \int_{B_{1}}\left(|\nabla \bar{u}|^{2}+\bar{u}^{2}\right) \mathrm{d} x-\frac{1}{q} \int_{B_{1}} b(x)|\bar{u}|^{q} \mathrm{~d} x \\
& \quad \geq \int_{B_{1}} a(|x|)|\bar{u}|^{p-2} \bar{u}(\bar{v}-\bar{u}) \mathrm{d} x \\
& =\int_{B_{1}} \nabla \bar{v} \cdot \nabla(\bar{v}-\bar{u}) \mathrm{d} x+\int_{B_{1}} \bar{v}(\bar{v}-\bar{u}) \mathrm{d} x+\int_{B_{1}} b(x)|\bar{v}|^{q-2} \bar{v}(\bar{v}-\bar{u}) \mathrm{d} x,
\end{aligned}
$$

where the last line follows from (16). Therefore,

$$
\begin{aligned}
& \frac{1}{2} \int_{B_{1}}|\nabla \bar{v}-\nabla \bar{u}|^{2} \mathrm{~d} x+\frac{1}{2} \int_{B_{1}}|\bar{v}-\bar{u}|^{2} \mathrm{~d} x \\
& \quad+\frac{1}{q} \int_{B_{1}} b(x)\left(|\bar{u}|^{q}-|\bar{v}|^{q}\right) \mathrm{d} x+\int_{B_{1}} b(x)|\bar{v}|^{q-2} \bar{v}(\bar{v}-\bar{u}) \mathrm{d} x \leq 0 .
\end{aligned}
$$

Note that the function $g: \mathbb{R} \rightarrow \mathbb{R}$ defined by $g(t)=|t|^{q} / q$ is convex. Thus for all $t_{1}, t_{2} \in \mathbb{R}$ we have that

$$
g\left(t_{2}\right)-g\left(t_{1}\right) \geq g^{\prime}\left(t_{1}\right)\left(t_{2}-t_{1}\right)=\left|t_{1}\right|^{q-2} t_{1}\left(t_{2}-t_{1}\right) .
$$

Substituting $t_{2}=\bar{u}$ and $t_{1}=\bar{v}$ in the latter inequality implies that

$$
\frac{1}{q}|\bar{u}|^{q}-\frac{1}{q}|\bar{v}|^{q} \geq|\bar{v}|^{q-2} \bar{v}(\bar{u}-\bar{v}) .
$$

Multiplying both sides by $b(x)$ and integrating over $B_{1}$ yield that

$$
\frac{1}{q} \int_{B_{1}} b(x)|\bar{u}|^{q} \mathrm{~d} x-\frac{1}{q} \int_{B_{1}} b(x)|\bar{v}|^{q} \mathrm{~d} x \geq \int_{B_{1}} b(x)|\bar{v}|^{q-2} \bar{v}(\bar{u}-\bar{v}) \mathrm{d} x .
$$

Now from (17) and (18), we obtain that

$$
\frac{1}{2} \int_{B_{1}}|\nabla \bar{v}-\nabla \bar{u}|^{2} \mathrm{~d} x+\frac{1}{2} \int_{B_{1}}|\bar{v}-\bar{u}|^{2} \mathrm{~d} x \leq 0,
$$

which implies that $\bar{u}=\bar{v}$. Taking into account that $\bar{u}=\bar{v}$ in (16), we have that $\bar{u}$ is a weak solution of

$$
-\Delta u+u=a(|x|)|u|^{p-2} u-b(x)|u|^{q-2} u,
$$

with the Neumann boundary condition. 
It is worth noting that condition $i i)$ in Theorem 3.1 indeed shows that the triple $(\Psi, K, \Phi)$ satisfies the point-wise invariance condition at $\bar{u}$ given in Definition 1.3. In fact, it corresponds to the case where $G=0$. This is why Theorem 3.1 is a very particular case of the general Theorem 1.4.

We shall need some preliminary results before proving our main results. We begin with the following elementary lemma.

Lemma 3.2 The following assertions hold:

1. There exists $C_{0}>0$ such that

$$
\|u\|_{L^{\infty}\left(B_{1}\right)} \leq C_{0}\|u\|_{H^{1}\left(B_{1}\right)}, \quad \forall u \in K .
$$

2. There exists a constant $C>0$ such that

$$
\|u\|_{H^{1}\left(B_{1}\right)} \leq\|u\|_{V} \leq C\|u\|_{H^{1}\left(B_{1}\right)}, \quad \forall u \in K .
$$

Proof Let $0<r<1$ and $B_{r}$ be a ball centered at the origin with radius $r$. It follows from the continuous embedding of $H^{1}\left(B_{1} \backslash B_{r}\right) \subseteq L^{\infty}\left(B_{1} \backslash B_{r}\right)$ that there exists a constant $C_{0}>0$ such that

$$
\|u\|_{L^{\infty}\left(B_{1}\right)}=\|u\|_{L^{\infty}\left(B_{1} \backslash B_{r}\right)} \leq C_{0}\|u\|_{H^{1}\left(B_{1} \backslash B_{r}\right)} \leq C_{0}\|u\|_{H^{1}\left(B_{1}\right)}, \quad(\forall u \in K) .
$$

This completes the proof of the first part. For the second part, we have that

$$
\begin{aligned}
\|u\|_{H^{1}\left(B_{1}\right)} \leq\|u\|_{V} & =\|u\|_{H^{1}\left(B_{1}\right)}+\|u\|_{L_{a}^{p}\left(B_{1}\right)}+\|u\|_{L_{b}^{q}\left(B_{1}\right)} \\
& \leq\|u\|_{H^{1}\left(B_{1}\right)}+\|u\|_{L^{\infty}\left(B_{1}\right)}\left(\|a\|_{L^{1}\left(B_{1}\right)}+\|b\|_{L^{1}\left(B_{1}\right)}\right) \leq C\|u\|_{H^{1}\left(B_{1}\right)},
\end{aligned}
$$

where $C=1+C_{0}\left(\|a\|_{L^{1}\left(B_{1}\right)}+\|b\|_{L^{1}\left(B_{1}\right)}\right)$.

Lemma 3.3 Let $\Omega$ be a bounded open domain in $\mathbb{R}^{N}$ and $q \geq 2$. Suppose that $f \in L^{\infty}(\Omega)$, $b \in L^{1}(\Omega)$ and $u \in H^{1}(\Omega) \cap L_{b}^{q}(\Omega)$ be such that

$$
\int_{\Omega} \nabla u \cdot \nabla \varphi \mathrm{d} x+\int_{\Omega} u \varphi \mathrm{d} x+\int_{\Omega} b(x)|u|^{q-2} u \varphi \mathrm{d} x=\int_{\Omega} f \varphi \mathrm{d} x, \quad \forall \varphi \in H^{1}(\Omega) \cap L_{b}^{q}(\Omega) .
$$

If the functions $f$ and $b$ are nonnegative, then there exists a constant $C>0$ independent of $u$ such that for a.e. $x \in \Omega$ we have

$$
0 \leq u(x) \leq C\|f\|_{L^{\infty}(\Omega)} .
$$

Proof Consider a function $\eta \in C^{1}(\mathbb{R})$ such that

(i) $\eta$ is strictly increasing on the interval $[0, \infty)$,

(ii) $\eta(t)=0, \quad \forall t \in(-\infty, 0]$.

(iii) both $\eta$ and its derivative $\eta^{\prime}$ are bounded.

We first show that $u(x) \geq 0$ for a.e. $x \in \Omega$. Set $\varphi=-\eta(-u)$. Note that $\varphi \in H^{1}(\Omega) \cap$ $L_{b}^{q}(\Omega)$, so by (20) we have

$$
\int_{\Omega}|\nabla u|^{2} \eta^{\prime}(-u) \mathrm{d} x-\int_{\Omega} u \eta(-u) \mathrm{d} x-\int_{\Omega} b(x)|u|^{q-2} u \eta(-u) \mathrm{d} x=-\int_{\Omega} f \eta(-u) \mathrm{d} x .
$$

Note that $f \geq 0$ and $\eta(-u) \geq 0$. Thus from (22) one can deduce that

$$
-\int_{\Omega}\left(u+b(x)|u|^{q-2} u\right) \eta(-u) \mathrm{d} x \leq 0 .
$$


On the other hand, for all $t \in \mathbb{R}$ and $x \in \Omega$, we have $\left(t+b(x)|t|^{q-2} t\right) \eta(t) \geq 0$. Therefore,

$$
-\left(u+b(x)|u|^{q-2} u\right) \eta(-u)=0, \quad \text { a.e. } \quad x \in \Omega .
$$

So by the definition of $\eta$, we get $u(x) \geq 0$ for a.e. $x \in \Omega$.

We now prove the second inequality in (21). Let $b_{0}=\inf _{x \in \Omega} b(x)$ and note that $b_{0} \geq 0$. Set $M=\|f\|_{L^{\infty}(\Omega)}$. Since the map $t \rightarrow t+b_{0}|t|^{q-2} t-M$ is strictly increasing, there exists $C>0$ such that $t+b_{0}|t|^{q-2} t-M \leq 0$ if and only if $t \leq \mathrm{CM}$. Set $\varphi=\eta(u-\mathrm{CM})$. It follows from (iii) that $\varphi \in H^{1}(\Omega) \cap L_{b}^{\bar{q}}(\Omega)$, so by (20) we have

$$
\begin{aligned}
& \int_{\Omega}|\nabla u|^{2} \eta^{\prime}(u-\mathrm{CM}) \mathrm{d} x+\int_{\Omega} u \eta(u-\mathrm{CM}) \mathrm{d} x+\int_{\Omega} b(x)|u|^{q-2} u \eta(u-\mathrm{CM}) \mathrm{d} x \\
& \quad=\int_{\Omega} f \eta(u-\mathrm{CM}) \mathrm{d} x,
\end{aligned}
$$

from which one has that

$$
\begin{aligned}
& \int_{\Omega}|\nabla u|^{2} \eta^{\prime}(u-\mathrm{CM}) \mathrm{d} x+\int_{\Omega}(u-M) \eta(u-\mathrm{CM}) \mathrm{d} x+\int_{\Omega} b(x)|u|^{q-2} u \eta(u-\mathrm{CM}) \mathrm{d} x \\
& \quad=\int_{\Omega}(f-M) \eta(u-\mathrm{CM}) \mathrm{d} x .
\end{aligned}
$$

Note that $f-M \leq 0$ and $\eta(u-\mathrm{CM}) \geq 0$. Thus from (23) one can deduce that

$$
\int_{\Omega}\left(u+b(x)|u|^{q-2} u-M\right) \eta(u-\mathrm{CM}) \mathrm{d} x \leq 0 .
$$

Since $u$ is nonnegative, it follows that

$$
\int_{\Omega}\left(u+b_{0}|u|^{q-2} u-M\right) \eta(u-\mathrm{CM}) \mathrm{d} x \leq \int_{\Omega}\left(u+g(x)|u|^{q-2} u-M\right) \eta(u-\mathrm{CM}) \mathrm{d} x \leq 0 .
$$

On the other hand, by the properties of the function $\eta$, we have

$$
\left(t+b_{0}|t|^{q-2} t-M\right) \eta(t-\mathrm{CM}) \geq 0, \quad \forall t \in \mathbb{R} .
$$

Therefore,

$$
\left(u+b_{0}|u|^{q-2} u-M\right) \eta(u-\mathrm{CM})=0, \quad \text { a.e. } \quad x \in \Omega .
$$

Thus again by using the definition of $\eta$ we obtain that $u(x) \leq \mathrm{CM}$ for a.e. $x \in \Omega$.

Lemma 3.4 The functional $E_{K}$ defined in (12) satisfies the (PS) compactness condition if either of the following assertions hold:

1. $p>q$;

2. If $p<q$ then $\left(a^{q} / b^{p}\right)^{\frac{1}{q-p}} \in L^{1}(0,1)$.

Proof Suppose that $\left\{u_{n}\right\}$ is a sequence in $K$ such that $E\left(u_{n}\right) \rightarrow c \in \mathbb{R}, \epsilon_{n} \rightarrow 0$ and

$$
\Psi_{K}(v)-\Psi_{K}\left(u_{n}\right)+\left\langle D \Phi\left(u_{n}\right), u_{n}-v\right\rangle \geq-\epsilon_{n}\left\|v-u_{n}\right\|_{V}, \quad \forall v \in V .
$$

We must show that $\left\{u_{n}\right\}$ has a convergent subsequence in $V$. Firstly, we prove that $\left\{u_{n}\right\}$ is bounded in $V$. To do this, we will consider two cases $p>q$ and $p<q$ separately.

Case 1, $p>q$ :

Note that since $E\left(u_{n}\right) \rightarrow c$, then for large values of $n$ we have

$$
\frac{1}{2}\left\|u_{n}\right\|_{H^{1}}^{2}+\frac{1}{q}\left\|u_{n}\right\|_{L_{b}^{q}}^{q}-\frac{1}{p} \int_{B_{1}} a(|x|)\left|u_{n}\right|^{p} \mathrm{~d} x \leq c+1 .
$$


Now consider the function $g(r)=r^{q}-p(r-1)-1$ on the interval $(1,+\infty)$. One can see easily that if we set $r^{*}=\left(\frac{p}{q}\right)^{\frac{q}{q-1}}$, then for every $r \in\left(1, r^{*}\right)$ we have $g(r)<0$. Choose such a number $r$. Thus, we have $r>1$ and $r^{q}-1<p(r-1)$. Note that

$$
\left\langle D \varphi\left(u_{n}\right), u_{n}\right\rangle=\int_{B_{1}} a(|x|) u_{n}(x)^{p} \mathrm{~d} x .
$$

Thus, by setting $v=r u_{n}$ in (24) we get

$$
\frac{\left(1-r^{2}\right)}{2}\left\|u_{n}\right\|_{H^{1}}^{2}+\frac{\left(1-r^{q}\right)}{q}\left\|u_{n}\right\|_{L_{b}^{q}}^{q}+(r-1) \int_{B_{1}} a(|x|)\left|u_{n}\right|^{p} \mathrm{~d} x \leq \epsilon_{n}(r-1)\left\|u_{n}\right\|_{V} \leq C\left\|u_{n}\right\|_{V} .
$$

Remember that $r^{q}-1<p(r-1)$, so take $\alpha>0$ such that

$$
\frac{1}{p(r-1)}<\alpha<\frac{1}{r^{q}-1} \text {. }
$$

Multiply (26) by $\alpha$ and sum it up with (25) to get

$$
\begin{aligned}
& \frac{1+\alpha\left(1-r^{2}\right)}{2}\left\|u_{n}\right\|_{H^{1}}^{2}+\frac{1+\alpha\left(1-r^{q}\right)}{q}\left\|u_{n}\right\|_{L_{b}^{q}}^{q} \\
& +\left[\alpha(r-1)-\frac{1}{p}\right] \int_{B_{1}} a(|x|)\left|u_{n}\right|^{p} \mathrm{~d} x \leq c+1+\alpha C\left\|u_{n}\right\|_{V} .
\end{aligned}
$$

Now from the choice of $\alpha$, the fact that $r>1$ and $q \geq 2$, all the coefficients in the left-hand side of the latter inequality are positive, thus for suitable constants $C_{1}, C_{2}, C_{3}, C_{4}>0$ we have that

$$
\left\|u_{n}\right\|_{H^{1}}^{2}+C_{1}\left\|u_{n}\right\|_{L_{b}^{q}}^{q}+C_{2} \int_{B_{1}} a(|x|)\left|u_{n}\right|^{p} \mathrm{~d} x \leq C_{3}+C_{4}\left\|u_{n}\right\|_{V} .
$$

As a consequence, by the second part of Lemma 3.2, one obtains that

$$
\left\|u_{n}\right\|_{H^{1}}^{2} \leq C_{3}+C_{4}\left\|u_{n}\right\|_{V} \leq C_{3}+C_{4} C\left\|u_{n}\right\|_{H^{1}}
$$

Therefore, $\left\{u_{n}\right\}$ is bounded in $H^{1}$. This completes the boundedness proof for the case $p>q$. Case 2, $p<q$ :

Let $u \in K$. By the Hölder inequality one has that

$$
\int_{B_{1}} a(|x|)|u|^{p} \mathrm{~d} x \leq\left\|a(b)^{\frac{-p}{q}}\right\|_{L^{\frac{q}{q-p}}}\left(\int_{B_{1}} b(|x|)|u|^{q} \mathrm{~d} x\right)^{\frac{p}{q}} .
$$

Thus

$$
\begin{aligned}
E_{K}(u) \geq & \frac{1}{2} \int_{B_{1}}\left(|\nabla u|^{2}+u^{2}\right) \mathrm{d} x+\frac{1}{q} \int_{B_{1}} b(|x|)|u|^{q} \mathrm{~d} x \\
& -\frac{1}{p}\left\|a(b)^{\frac{-p}{q}}\right\|_{L^{\frac{q}{q-p}}}\left(\int_{B_{1}} b(|x|)|u|^{q} \mathrm{~d} x\right)^{\frac{p}{q}} \\
= & \frac{1}{2}\|u\|_{H^{1}}^{2}+\frac{1}{q}\|u\|_{L_{b}^{q}}^{q}-\frac{1}{p}\left\|a(b)^{\frac{-p}{q}}\right\|_{L^{\frac{q}{q-p}}}\|u\|_{L_{b}^{q}}^{p} .
\end{aligned}
$$

Since $p<q$ and $\left\|a(b)^{\frac{-p}{q}}\right\|_{L^{\frac{q}{q-p}}}<\infty$, the latter inequality implies that $E_{K}$ is bounded from below and coercive on $V$ taking into consideration the second part of Lemma 3.2. This indeed implies that the sequence $\left\{u_{n}\right\}$ must also be bounded in $V$ for the case $p<q$. 
Therefore, for both cases $p>q$ and $p<q$ we have the boundedness of $\left\{u_{n}\right\}$ in $V$. Using standard results in Sobolev spaces, after passing to a subsequence if necessary, there exists $\bar{u} \in V$ such that $u_{n} \rightarrow \bar{u}$ weakly in $H^{1}, u_{n} \rightarrow \bar{u}$ strongly in $L^{2}$ and $u_{n} \rightarrow \bar{u}$ a.e.. We also have the weak convergence of $u_{n}$ to $\bar{u}$ in both $L_{b}^{q}$ and $L_{a}^{p}$. Also according to Lemma 3.2 from boundedness of $\left\{u_{n}\right\} \subset K$ in $H^{1}$ one can deduce that $\left\{u_{n}\right\}$ is bounded in $L^{\infty}$. Note that every $u_{n}$ is radial, so $\bar{u}$ is radial too, and moreover, $\bar{u} \in K$.

It follows from the properties of weak convergence that

$$
\begin{aligned}
\|\bar{u}\|_{H^{1}} & \leq \liminf _{n \rightarrow \infty}\left\|u_{n}\right\|_{H^{1}}, \\
\|\bar{u}\|_{L_{b}^{q}} & \leq \liminf _{n \rightarrow \infty}\left\|u_{n}\right\|_{L_{b}^{q}} .
\end{aligned}
$$

Now in (24) set $v=\bar{u}$ :

$\frac{1}{2}\left(\|\bar{u}\|_{H^{1}}^{2}-\left\|u_{n}\right\|_{H^{1}}^{2}\right)+\frac{1}{q}\left(\|\bar{u}\|_{L_{b}^{q}}^{q}-\left\|u_{n}\right\|_{L_{b}^{q}}^{q}\right)+\int_{B_{1}} a(|x|)\left|u_{n}\right|^{p-2} u_{n}\left(u_{n}-\bar{u}\right) \mathrm{d} x \geq-\epsilon_{n}\left\|u_{n}-\bar{u}\right\|_{V}$.

Since $\left\|u_{n}\right\|_{L^{\infty}}$ is bounded, one has that

$$
a(|x|)\left|u_{n}\right|^{p-1}\left|\bar{u}(x)-u_{n}(x)\right| \leq a(|x|)\left\|u_{n}\right\|_{L^{\infty}}^{p-1}\left(\left\|u_{n}\right\|_{L^{\infty}}+\|\bar{u}\|_{L^{\infty}}\right) \leq \tilde{C} a(|x|),
$$

and

$$
a(|x|)\left|u_{n}\right|^{p} \leq a(|x|)\left\|u_{n}\right\|_{L^{\infty}}^{p} \leq \tilde{C} a(|x|),
$$

for some constant $\tilde{C}>0$. Since $a \in L^{1}$, it follows from dominated convergence theorem that

$$
\lim _{n \rightarrow \infty} \int_{B_{1}} a(|x|)\left|u_{n}\right|^{p-2} u_{n}\left(\bar{u}-u_{n}\right) \mathrm{d} x=0, \quad \& \quad \lim _{n \rightarrow \infty} \int_{B_{1}} a(|x|)\left|u_{n}\right|^{p} \mathrm{~d} x=\int_{B_{1}} a(|x|)|u|^{p} \mathrm{~d} x .
$$

Therefore, it follows from (30) and (31) that

$$
\frac{1}{2}\left(\limsup _{n \rightarrow \infty}\left\|u_{n}\right\|_{H^{1}}^{2}-\|\bar{u}\|_{H^{1}}^{2}\right)+\frac{1}{q}\left(\limsup _{n \rightarrow \infty}\left\|u_{n}\right\|_{L_{b}^{q}}^{q}-\|\bar{u}\|_{L_{b}^{q}}^{q}\right) \leq 0 .
$$

Hence, by taking into account the inequalities in (29) we obtain that

$$
\|\bar{u}\|_{H^{1}}^{2}=\lim _{n \rightarrow \infty}\left\|u_{n}\right\|_{H^{1}}, \quad \& \quad \lim _{n \rightarrow \infty}\left\|u_{n}\right\|_{L_{b}^{q}}^{q}=\|\bar{u}\|_{L_{b}^{q}}^{q} .
$$

The latter together with (31) yield that

$$
u_{n} \rightarrow \bar{u} \text { strongly in } \quad V
$$

as desired.

To prove the second condition in Theorem 3.1, we shall need the following result for which a proof already exists in [15]. However, for the convenience of the reader we shall also provide a sketch for the proof in the present paper.

Lemma 3.5 Let $h \in L^{1}(0,1)$ be a nonnegative monotone function. Then there exists a sequence of smooth monotone functions $\left\{h_{m}\right\}$ with the property that $0 \leq h_{m} \leq h$ and $h_{m} \rightarrow h$ strongly in $L^{1}(0,1)$. 
Proof We do the proof for the case where the function $h$ is non-decreasingly monotone. The same argument works if $h$ is non-increasingly monotone. For large integers $m$ we define $q_{m}$ on $[0, \infty)$ via $q_{m}(r)=\min \{h(r), m\}$ and so note for each $m$ that $q_{m}$ is non-decreasing on $(0,1)$. Now extend $q_{m}(r)$ to $q_{m}(1)$ for $r>1$ and $q_{m}=0$ for $r<0$. Let $0 \leq \eta$ be smooth with $\eta=0$ on $(-\infty,-1) \cup(0, \infty)$ and $\eta>0$ on $(-1,0)$. We also assume that $\int_{-1}^{0} \eta(\tau) d \tau=1$. For $\epsilon>0$, define $\eta_{\epsilon}(r):=\frac{1}{\epsilon} \eta\left(\frac{r}{\epsilon}\right)$ and

$$
q_{m}^{\epsilon}(r):=\int_{-\epsilon}^{0} \eta_{\epsilon}(\tau) q_{m}(r+\tau) d \tau,
$$

note that this is just the usual mollification except the support of $\eta$ is adjusted slightly. Since $q_{m}$ is non-decreasing, we see that for each fixed small $\epsilon>0$ that $q_{m}^{\epsilon}$ is non-decreasing in $r$. Then note that we have

$$
0 \leq q_{m}^{\epsilon}(r)=\int_{-\epsilon}^{0} \eta_{\epsilon}(\tau) q_{m}(r+\tau) d \tau \leq q_{m}(r) \int_{-\epsilon}^{0} \eta_{\epsilon}(\tau) d \tau=q_{m}(r) \leq h(r) .
$$

We now let $\epsilon_{m} \searrow 0$ and we set $h_{m}(r):=q_{m}^{\epsilon_{m}}$. So we have $0 \leq h_{m}(r) \leq h(r)$ for all $m$. Also $r \mapsto h_{m}(r)$ is non-decreasing in $r$. One can now show that $h_{m} \rightarrow h$ strongly in $L^{1}(0,1)$.

Lemma 3.6 Suppose $\bar{u} \in K$. Then there exists $v \in K$ satisfying

$$
\left\{\begin{array}{lr}
-\Delta v+v+b(x)|v|^{q-2} v=a(|x|)|\bar{u}|^{p-2} \bar{u}, & x \in B_{1} \\
\frac{\partial v}{\partial v}=0, & x \in \partial B_{1},
\end{array}\right.
$$

in the weak sense.

Proof By Lemma 3.5, there exists a sequence $\left\{a_{m}\right\}$ (resp. $\left\{b_{m}\right\}$ ) of smooth functions such that $0 \leq a_{m} \leq a$ (resp. $0 \leq b_{m} \leq b$ ) and each $a_{m}$ is non-decreasing (resp. each $b_{m}$ is non-increasing) on $(0,1)$ and $a_{m} \rightarrow a$ in $L^{1}(0,1)$ (resp. $b_{m} \rightarrow b$ in $L^{1}(0,1)$ ). We shall consider the following problem.

$$
\left\{\begin{array}{lr}
-\Delta v+v+b_{m}|v|^{q-2} v=a_{m}(|x|)|\bar{u}|^{p-2} \bar{u}, & x \in B_{1} \\
\frac{\partial v}{\partial v}=0, & x \in \partial B_{1},
\end{array}\right.
$$

Set $f_{m}(x)=a_{m}(|x|)|\bar{u}|^{p-2} \bar{u}$. Note that $f_{m} \in H^{1}$ as $a_{m}$ is smooth and $\bar{u} \in K$. Here we have used the fact that $K \subset L^{\infty}$ by virtue of Lemma 3.2. Now define

$$
J(v):=\frac{1}{2} \int_{B_{1}}\left(|\nabla v|^{2}+|v|^{2}\right) \mathrm{d} x+\frac{1}{q} \int_{B_{1}} b_{m}|v|^{q} \mathrm{~d} x-\int_{B_{1}} f_{m}(x) v \mathrm{~d} x,
$$

on the space $H_{\text {rad }}^{1}$. Observe that $J$ is convex and lower semi-continuous. Also

$$
\lim _{\|v\|_{H^{1}} \rightarrow \infty} J(v)=\infty
$$

therefore $J$ takes its minimum at some $v_{m} \in H_{\text {rad }}^{1}$. It is also easily seen that $\frac{\partial v_{m}}{\partial v}=0$. We want to show that $v_{m} \in K$. Note first that $v_{m} \in H^{1}\left(B_{1}\right) \cap L_{b_{m}}^{q}$ satisfies the following equation

$$
\begin{aligned}
& \int_{B_{1}} \nabla v_{m} . \nabla \varphi \mathrm{d} x+\int_{B_{1}} v_{m} \varphi \mathrm{d} x+\int_{B_{1}} b_{m}\left|v_{m}\right|^{q-2} v_{m} \varphi \mathrm{d} x \\
& =\int_{B_{1}} f_{m} \varphi \mathrm{d} x, \quad \forall \varphi \in H^{1}\left(B_{1}\right) \cap L_{b_{m}}^{q}\left(B_{1}\right) .
\end{aligned}
$$


Therefore, by Lemma 3.3 together with the fact that $f_{m} \geq 0$ we obtain that $v_{m} \geq 0$, a.e. in $B_{1}$.

Now we need to prove some regularity for $v_{m}$. Note that $a_{m}$ is smooth and $\bar{u} \in K$, so by Lemma 3.2 one has that $f_{m} \in L^{\infty}\left(B_{1}\right)$. Thus from Lemma 3.3, we can deduce that $v_{m} \in L^{\infty}\left(B_{1}\right)$. Now $v_{m} \in H^{1}\left(B_{1}\right)$ is a weak solution of the following equation

$$
-\Delta v_{m}+v_{m}=f_{m}-b_{m}\left|v_{m}\right|^{q-2} v_{m}:=g_{m},
$$

and observe that $g_{m} \in L^{2}$ since $v_{m} \in L^{\infty}$ and $b_{m}$ is smooth. Also $\nabla g_{m} \in L^{2}\left(B_{1}\right)$ as the functions $a_{m}, b_{m}, v_{m} \in H^{1}$ and $v_{m} \in L^{\infty}$. Thus $g_{m} \in H^{1}\left(B_{1}\right)$. Now by standard regularity theory one can deduce that $v_{m} \in H^{3}\left(B_{1}\right)$.

It remains to show that the radial function $v_{m}$ is non-decreasing in $r=|x|$. For $0<r<1$, set $w_{m}(x):=\left(v_{m}\right)_{r}(|x|)$, the derivative of $v_{m}$ with respect to $r=|x|$. Thus, $w_{m}$ satisfies

$$
\begin{cases}-\Delta w_{m}+\left(\frac{N-1}{|x|^{2}}+1+(q-1) b_{m}\left|v_{m}\right|^{q-2}\right) w_{m}=Q_{m}(x), & x \in B_{1} \backslash\{0\} \\ w_{m}=0, & x \in \partial B_{1},\end{cases}
$$

where

$$
Q_{m}(x):=a_{m}^{\prime}(r) \bar{u}(r)^{p-1}+a_{m}(r)(p-1) \bar{u}(r)^{p-2} \bar{u}^{\prime}(r)-b_{m}^{\prime}\left|v_{m}\right|^{q-2} v_{m},
$$

on $(0,1)$. Since $a_{m}$ and $\bar{u}$ are nonnegative and non-decreasing, the first two terms in $Q_{m}$ are nonnegative. Also $v_{m}$ is nonnegative and $b_{m}$ is non-increasing which makes the last term in $Q_{m}$ nonnegative. Thus, $Q_{m} \geq 0$ on $(0,1)$. Note that $w_{m} \in H_{\mathrm{rad}}^{1}\left(B_{1}\right)$ and has enough regularity to extend the solution of (36) to the full ball $B_{1}$. If we define the linear operator $L$ as

$$
L\left(w_{m}\right):=-\Delta w_{m}+\left(\frac{N-1}{|x|^{2}}+1+(q-1) b_{m}\left|v_{m}\right|^{q-2}\right) w_{m},
$$

then $L\left(w_{m}\right) \geq 0$. We now show that $w_{m} \geq 0$ in $B_{1}$. Note that $w_{m}=w_{m}^{+}-w_{m}^{-}$where $w_{m}^{+}=\max \left\{0, w_{m}(x)\right\}$ and $w_{m}^{-}=\max \left\{0,-w_{m}(x)\right\}$. Multiplying the inequality $L\left(w_{m}\right) \geq 0$ by $-w_{m}^{-}$and integrating over $B_{1}$ implies that

$$
\int_{B_{1}}\left(\left|\nabla w_{m}^{-}\right|^{2}+\left|w_{m}^{-}\right|^{2}\right) \mathrm{d} x \leq 0
$$

from which we obtain that $w_{m}^{-}=0$. Thus, $w_{m} \geq 0$ and consequently $v_{m}$ is non-decreasing in $r$. Therefore, $v_{m} \in K$.

We now show that $\left\{v_{m}\right\}$ is bounded in $V$. It follows from (35) with $\varphi=v_{m}$ that

$$
\int_{B_{1}}\left(\left|\nabla v_{m}\right|^{2}+\left|v_{m}\right|^{2}+b_{m}\left|v_{m}\right|^{q}\right) \mathrm{d} x=\int_{B_{1}} f_{m} v_{m} \mathrm{~d} x .
$$

On the other hand,

$$
\begin{aligned}
\int_{B_{1}} f_{m} v_{m} \mathrm{~d} x & =\int_{B_{1}} a_{m}(|x|)|\bar{u}|^{p-2} \bar{u} v_{m} \mathrm{~d} x \leq \int_{B_{1}} a(|x|)|\bar{u}|^{p-2} \bar{u} v_{m} \mathrm{~d} x \\
& \leq\|\bar{u}\|_{L^{\infty}}^{p-1}\|a\|_{L^{1}}\left\|v_{m}\right\|_{L^{\infty}} \leq C_{1}\left\|v_{m}\right\|_{H^{1}},
\end{aligned}
$$

where $C_{1}$ is a constant independent of $m$. Here we have used Lemma 3.2 together with the fact that $a \in L^{1}(0,1)$ and $\bar{u}, v_{m} \in K$. It now follows from (37) and (38) that

$$
\left\|v_{m}\right\|_{H^{1}}^{2}+\int_{B_{1}} b_{m}\left|v_{m}\right|^{q} \mathrm{~d} x \leq C_{1}\left\|v_{m}\right\|_{H^{1}}
$$


from which the boundedness of $\left\|v_{m}\right\|_{H^{1}}^{2}$ and $\int_{B_{1}} b_{m}\left|v_{m}\right|^{q} \mathrm{~d} x$ follows. By Lemma 3.2, we obtain that $\left\|v_{m}\right\|_{L^{\infty}}$ is also bounded. By passing to a subsequence, we can assume there is $0 \leq v \in H_{\text {rad }}^{1}\left(B_{1}\right)$ such that $v_{m} \rightarrow v$ a.e. and $v_{m} \rightarrow v$ weakly in $H^{1}\left(B_{1}\right)$. Also $v$ is nondecreasing in $r$ on $(0,1)$ since each $v_{m}$ has this property. Thus, by Lemma 3.2, $v \in L^{\infty}$ from which we obtain particularly that $v \in K$. It remains to show that $v$ satisfies Eq. (33). Suppose $\eta \in H^{1}\left(B_{1}\right) \cap L^{\infty}\left(B_{1}\right)$, then from (35) we get

$$
\int_{B_{1}}\left(\nabla v_{m} \nabla \eta+v_{m} \eta+\left|v_{m}\right|^{q-2} v_{m} \eta\right) \mathrm{d} x=\int_{B_{1}} a_{m}|\bar{u}|^{p-2} \bar{u} \eta \mathrm{d} x .
$$

We show that

$$
\int_{B_{1}} b_{m}\left|v_{m}\right|^{q-2} v_{m} \eta \mathrm{d} x \rightarrow \int_{B_{1}} b|v|^{q-2} v \eta \mathrm{d} x .
$$

It follows from the boundedness of $\left\|v_{m}\right\|_{L^{\infty}}$ that

$$
\left.\left|b_{m}\right| v_{m}\right|^{q-2} v_{m} \eta \mid \leq b\left\|v_{m}\right\|_{L^{\infty}}^{q-1}\|\eta\|_{L^{\infty}} \leq C_{2} b,
$$

for some positive constant $C_{2}$. Thus, the relation (41) follows from the dominated convergence theorem. Therefore, by passing to the limit in (40) we get that

$$
\int_{B_{1}}\left(\nabla v \nabla \eta+v \eta+b(x)|v|^{q-2} v \eta\right) \mathrm{d} x=\int_{B_{1}} a|\bar{u}|^{p-2} \bar{u} \eta \mathrm{d} x .
$$

By density, the latter holds for all $\eta \in H^{1}\left(B_{1}\right) \cap L_{b}^{q}\left(B_{1}\right)$. This means that $v$ is a solution of (33) in the weak sense.

Proof of Theorem 1.1 First, we shall use Theorem 2.3 to prove the existence of a non-trivial critical point for $E_{K}$. Since by Lemma 3.4 the functional $E_{K}$ satisfies the (PS) compactness condition, we just need to verify the mountain pass geometry of the functional $E_{K}$.

It is clear that $E_{K}(0)=0$. Take $e \in K$. It follows that

$$
E_{K}(t e)=\frac{t^{2}}{2} \int_{B_{1}}\left(|\nabla e|^{2}+|e|^{2}\right) \mathrm{d} x+\frac{t^{q}}{q} \int_{B_{1}} b(|x|)|e|^{q} \mathrm{~d} x-\frac{t^{p}}{p} \int_{B_{1}} a(|x|)|e|^{p} \mathrm{~d} x
$$

Now, since $p>q \geq 2$, thus for $t$ sufficiently large $E_{K}(t e)$ is negative.

We now prove condition (3) of $(M P G)$. Take $u \in K$ with $\|u\|_{V}=\rho>0$. We have

$$
E_{K}(u)=\frac{1}{2}\|u\|_{H^{1}}^{2}+\frac{1}{q}\|u\|_{L_{b}^{q}}^{q}-\frac{1}{p} \int_{B_{1}} a(|x|)|u|^{p} \mathrm{~d} x .
$$

Note that from Lemma 3.2, there exist positive constant $C$ such that for every $u \in K$ one has

$$
\|u\|_{H^{1}} \leq\|u\|_{V} \leq C\|u\|_{H^{1}} .
$$

We also have that

$$
\int_{B_{1}} a(|x|)|u|^{p} \mathrm{~d} x \leq\|u\|_{V}^{p}
$$

Therefore,

$$
\begin{aligned}
E_{K}(u) & \geq \frac{1}{2}\|u\|_{H^{1}}^{2}-\frac{1}{p} \int_{B_{1}} a(|x|)|u|^{p} \mathrm{~d} x \geq \frac{1}{2}\|u\|_{H^{1}}^{2}-\frac{1}{p}\|u\|_{V}^{p} \\
& \geq \frac{1}{2 C^{2}}\|u\|_{V}^{2}-\frac{1}{p}\|u\|_{V}^{p}=\frac{1}{2 C^{2}} \rho^{2}-\frac{1}{p} \rho^{p}>0,
\end{aligned}
$$


provided $\rho>0$ is small enough, since $p>2$. If $u \notin K$, then clearly $E_{K}(u)>0$. Thus, MPG holds for the functional $E_{K}$. Therefore, the functional $E_{K}$ has a non-trivial critical point $\bar{u} \in K$. It also follows from Lemma 3.6 that there exists $v \in K$ which satisfies the equation in the weak sense

$$
\left\{\begin{array}{lrl}
-\Delta v+v+b(x)|v|^{q-2} v=a(|x|)|\bar{u}|^{p-2} \bar{u}, & x \in B_{1} \\
\frac{\partial v}{\partial v}=0, & x \in \partial B_{1},
\end{array}\right.
$$

It now follows from Theorem 3.1 that $\bar{u}$ is indeed a solution of (1) with $E_{K}(\bar{u})>0$.

In the following theorem, we show that a similar result to Theorem 1.1 also holds for the case where $p<q$.

Theorem 3.7 Let $p$ and $q$ be two real numbers $2 \leq p<q$. Assume that the functions a and $b$ satisfy the following conditions:

H1. $a \in L^{1}(0,1)$ is non-decreasing and $a(r)>0$ for a.e. $r \in[0,1]$.

$H 2 . \quad b \in L^{1}(0,1)$ is nonnegative and non-increasing on $[0,1]$.

H3. $\left(a^{q} / b^{p}\right)^{\frac{1}{q-p}} \in L^{1}(0,1)$ and there exists $e \in K$ such that $E_{K}(e)<0$.

Then problem (1) admits at least one radially non-decreasing positive solution.

Proof Set $\mu=\inf _{V} E(u)$. It follows from (28) that $E_{K}$ is bounded from below. By Lemma 3.4, the functional $E_{K}$ satisfies the (PS) compactness condition. Thus, it follows from Theorem 2.4 that $\min _{u \in V} E_{K}(u)$ is attained at some $\bar{u} \in K$ which is indeed a critical point of $E_{K}$.

It also follows from Lemma 3.6 that there exists $v \in K$ which satisfies the equation in the weak sense

$$
\begin{cases}-\Delta v+v+b(x)|v|^{q-2} v=a(|x|)|\bar{u}|^{p-2} \bar{u}, & x \in B_{1} \\ \frac{\partial v}{\partial v}=0, & x \in \partial B_{1},\end{cases}
$$

Thus, by Theorem 3.1, $\bar{u}$ is a solution of (1). Note also that

$$
E_{K}(\bar{u})=\min _{u \in V} E_{K}(u) \leq E_{K}(e)<0,
$$

and therefore, $\bar{u}$ is non-trivial.

\section{Non-constant solutions}

In this section, we consider the problem

$$
\begin{cases}-\Delta u+u=a|u|^{p-2} u-b|u|^{q-2} u, & x \in B_{1}, \\ u>0, & x \in B_{1}, \\ \frac{\partial u}{\partial v}=0, & x \in \partial B_{1},\end{cases}
$$

where $a>0$ and $b>0$ are two constants. In Theorem 1.1, we have proved that this problem has a nonzero solution when $p>q \geq 2$. However, any nonzero constant function $u$ satisfying

$$
Q(u):=a|u|^{p-2} u-b|u|^{q-2} u-u=0,
$$

is also a nonzero solution. In the following theorem, by adapting an argument from [5,7], we show that for certain values of $p$ and $q$ the solution obtained in Theorem 1.1 is indeed 
non-constant. We also refer to the interesting paper [10] where more general non-linearities are considered and a generalization to higher eigenvalues is provided.

We need the following lemma which states some simple properties of the eigenfunction associated with $\lambda_{2}$, the second radial eigenvalue of $-\Delta+1$ in the unit ball with Neumann boundary conditions.

Lemma 4.1 Let $v$ be an eigenfunction associated with $\lambda_{2}$, that is, a non-trivial solution of

$$
\begin{cases}-\Delta v+v=\lambda_{2} v, & x \in B_{1}, \\ \frac{\partial v}{\partial v}=0, & x \in \partial B_{1} .\end{cases}
$$

Then $\lambda_{2}>1$, $v$ is radial and unique up to a multiplicative factor and we can choose it increasing. Moreover, $\int_{B_{1}} v \mathrm{~d} x=0$.

We refer to ([7], Lemma 4.8) for the proof of Lemma 4.1. Here is our result for the existence of non-constant solutions.

Theorem 4.2 Suppose that $2<q<p$. Also assume $a \equiv 1, b>0$ and $b(q-2)<p-2$. Then there exists at least one non-constant non-decreasing radial solution of (1).

Proof First we show that the function $Q$ has a unique nonzero root. Set $h(t):=t^{p-2}-$ $b t^{q-2}-1$. Note that $h(1)=-b<0$ and $\lim _{t \rightarrow+\infty} h(t)=+\infty$. Thus, $h(t)=0$ has a root in the interval $(1,+\infty)$. Also $h^{\prime}(t)=t^{q-3}\left[(p-2) t^{p-q}-b(q-2)\right]>0$ in the interval $(1,+\infty)$ as $b(q-2)<p-2$. Thus, $h$ is strictly increasing in the interval $(1,+\infty)$ which means that $h$ has a unique root in this interval. On the other hand, in the interval $[0,1]$ we have $h(t)<0$. Therefore, problem (43) has a unique constant solution. Denote this solution by $\bar{u}$ and note that $\bar{u} \in(1,+\infty)$.

Now we show that the solution obtained in Theorem 1.1 is different from $\bar{u}$. Recall that problem (1) has a positive solution $u$ with $E(u)=E_{K}(u)=c>0$ where the critical value $c$ is characterized by

$$
c=\inf _{\gamma \in \Gamma} \max _{t \in[0,1]} E_{K}[\gamma(t)]
$$

where

$$
\Gamma=\left\{\gamma \in C([0,1], V): \gamma(0)=0 \neq \gamma(1), E_{K}(\gamma(1)) \leq 0\right\} .
$$

We shall show that $E(u)=c<E(\bar{u})$. It then implies that $u$ is non-constant. Let $v$ be as in Lemma 4.1, and $s>0$ be such that $s<\|\bar{u}\|_{\infty} /\|v\|_{\infty}$. Thus, $\bar{u}+s v \in K$. For $r \in \mathbb{R}$ we have

$$
E[(\bar{u}+s v) r]=\frac{r^{2}}{2} \int_{B_{1}}\left(\bar{u}+s \sqrt{\lambda_{2}} v\right)^{2} \mathrm{~d} x+\frac{r^{q}}{q} \int_{B_{1}} b(\bar{u}+s v)^{q} \mathrm{~d} x-\frac{r^{p}}{p} \int_{B_{1}}(\bar{u}+s v)^{p} \mathrm{~d} x .
$$

Note that $q<p$, so there exists $r>1$ such $E[(\bar{u}+s v) r] \leq 0$. Set $\gamma_{s}(t)=t(\bar{u}+s v) r$. Note that $\gamma_{s} \in \Gamma$. We shall show that there exists $s>0$ such that for every $t \in[0,1]$ one has $E\left(\gamma_{s}(t)\right)<E(\bar{u})$. Therefore,

$$
c \leq \max _{t \in[0,1]} E\left(\gamma_{s}(t)\right)<E(\bar{u}) .
$$

Define $\xi: \mathbb{R}^{2} \rightarrow \mathbb{R}$ by

$$
\xi(s, t):=E^{\prime}(t(\bar{u}+s v) r)(\bar{u}+s v) r .
$$


Note that $\xi$ is $C^{1}$ and $\xi(0,1 / r)=0$. Also

$$
\begin{aligned}
\left.\frac{\mathrm{d}}{\mathrm{d} t}\right|_{(0,1 / r)} \xi(s, t) & =E^{\prime \prime}(\bar{u})(r \bar{u}, r \bar{u}) \\
& =r^{2} \int_{B_{1}}\left(1+b(q-1) \bar{u}^{q-2}-(p-1) \bar{u}^{p-2}\right) \bar{u}^{2} \mathrm{~d} x \\
& =r^{2} \int_{B_{1}} \bar{u}^{p}-b \bar{u}^{q}+b(q-1) \bar{u}^{q}-(p-1) \bar{u}^{p} \mathrm{~d} x \\
& =r^{2} \int_{B_{1}} b(q-2) \bar{u}^{q}-(p-2) \bar{u}^{p} \mathrm{~d} x<0,
\end{aligned}
$$

where we have used the fact that $Q(\bar{u})=0, \bar{u} \geq 1$ and $b(q-2)<p-2$. It follows from the implicit function theorem that there exist $\epsilon_{1}, \epsilon_{2}>0$ and a $C^{1}$ function $g:\left(-\epsilon_{1}, \epsilon_{1}\right) \rightarrow$ $\left(1 / r-\epsilon_{2}, 1 / r+\epsilon_{2}\right)$ such that $g(0)=1 / r$ and for $(s, t) \in\left(-\epsilon_{1}, \epsilon_{1}\right) \times\left(1 / r-\epsilon_{2}, 1 / r+\epsilon_{2}\right)$ one has $\xi(s, t)=0$ iff $t=g(s)$. Note also that

$$
\begin{aligned}
\left.\frac{\mathrm{d}}{\mathrm{d} s}\right|_{(0,1 / r)} \xi(s, t) & =E^{\prime \prime}(\bar{u})(\bar{u}, r v)+E^{\prime}(\bar{u}) r v \\
& =r \int_{B_{1}}\left(1+b(q-1) \bar{u}^{q-2}-(p-1) \bar{u}^{p-2}\right) \bar{u} v \mathrm{~d} x \\
& =r\left(1+b(q-1) \bar{u}^{q-2}-(p-1) \bar{u}^{p-2}\right) \bar{u} \int_{B_{1}} v \mathrm{~d} x=0
\end{aligned}
$$

where we have used the fact that $E^{\prime}(\bar{u})=0$ and $\int_{B_{1}} v \mathrm{~d} x=0$. Therefore, $g^{\prime}(0)=0$. Now we claim that

$$
E(g(s)(\bar{u}+s v) r)<E(\bar{u}), \quad \forall s \in\left(-\epsilon_{1}, \epsilon_{1}\right) .
$$

For this, note that since $g^{\prime}(0)=0$, then for every $s \in\left(-\epsilon_{1}, \epsilon_{1}\right)$ one has $g(s)=1 / r+o(s)$. Thus

$$
g(s)(\bar{u}+s v) r-\bar{u}=s(1 / r+o(s)) r v+\bar{u}(r g(s)-1)=s v+o(s) .
$$

Hence, by using the fact that $E^{\prime}(\bar{u})=0$ one can deduce that

$$
\begin{aligned}
E(g(s)(\bar{u}+s v) r)-E(\bar{u}) & =\frac{1}{2} E^{\prime \prime}(\bar{u})(g(s)(\bar{u}+s v) r-\bar{u}, g(s)(\bar{u}+s v) r-\bar{u})+o\left(s^{2}\right) \\
& =\frac{1}{2} E^{\prime \prime}(\bar{u})(s v+o(s), s v+o(s))+o\left(s^{2}\right) \\
& =\frac{s^{2}}{2} E^{\prime \prime}(\bar{u})(v, v)+o\left(s^{2}\right) .
\end{aligned}
$$


It then follows that

$$
\begin{aligned}
E & (g(s)(\bar{u}+s v) r)-E(\bar{u}) \\
& =\frac{s^{2}}{2} \int_{B_{1}}|\nabla v|^{2}+|v|^{2}+b(q-1) \bar{u}^{q-2} v^{2}-(p-1) \bar{u}^{p-2} v^{2} \mathrm{~d} x+o\left(s^{2}\right) \\
& =\frac{s^{2}}{2} \int_{B_{1}} \lambda_{2} v^{2}+b(q-1) \bar{u}^{q-2} v^{2}-(p-1) \bar{u}^{p-2} v^{2} \mathrm{~d} x+o\left(s^{2}\right) \\
& =\frac{s^{2}}{2 \bar{u}^{2}} \int_{B_{1}}\left(\lambda_{2} \bar{u}^{2}+b(q-1) \bar{u}^{q}-(p-1) \bar{u}^{p}\right) v^{2} \mathrm{~d} x+o\left(s^{2}\right) \\
& =\frac{s^{2}}{2 \bar{u}^{2}} \int_{B_{1}}\left(\lambda_{2} \bar{u}^{p}-\lambda_{2} b \bar{u}^{q}+b(q-1) \bar{u}^{q}-(p-1) \bar{u}^{p}\right) v^{2} \mathrm{~d} x+o\left(s^{2}\right),
\end{aligned}
$$

where we have used the identity $Q(\bar{u})=0$ in the last line. Therefore,

$$
\begin{aligned}
E(g(s)(\bar{u}+s v) r)-E(\bar{u})= & \frac{s^{2}}{2 \bar{u}^{2}} \int_{B_{1}}\left(b\left(q-1-\lambda_{2}\right) \bar{u}^{q}-\left(p-1-\lambda_{2}\right) \bar{u}^{p}\right) v^{2} \mathrm{~d} x \\
& +o\left(s^{2}\right)<0,
\end{aligned}
$$

where the last inequity follows from the fact that

$$
b \leq \frac{p-2}{q-2}<\frac{p-1-\lambda_{2}}{q-1-\lambda_{2}}
$$

considering $\lambda_{2}>1$. Now we claim that the function $t \mapsto E\left(\gamma_{0}(t)\right)$ has a unique maximum point at $t=1 / r$. For this, observe that

$$
\begin{aligned}
\frac{\mathrm{d}}{\mathrm{d} t} E\left(\gamma_{0}(t)\right) & =\frac{\mathrm{d}}{\mathrm{d} t} E(t \bar{u} r)=E^{\prime}(t \bar{u} r) r \bar{u} \\
& =\left|B_{1}\right|\left(t \bar{u}^{2} r^{2}+b t^{q-1} \bar{u}^{q} r^{q}-t^{p-1} \bar{u}^{p} r^{p}\right) \bar{u}=-\left|B_{1}\right| t \bar{u}^{2} r^{2} h(t \bar{u} r),
\end{aligned}
$$

where $\left|B_{1}\right|$ is te measure of the ball $B_{1}$. Thus $\frac{\mathrm{d}}{\mathrm{d} t} E\left(\gamma_{0}(t)\right)>0$ if $t<1 / r$ and $\frac{\mathrm{d}}{\mathrm{d} t} E\left(\gamma_{0}(t)\right)<0$ if $t>1 / r$. Thus $E\left(\gamma_{0}(t)\right)$ has a unique maximum point at $t=1 / r$.

By continuity of the function $(t, s) \rightarrow E\left(\gamma_{s}(t)\right)$ we can choose $0<s_{0}<\epsilon_{1}$ small enough such that the maximum of the function $t \rightarrow E\left(\gamma_{s_{0}}(t)\right)$ lies in the interval $\left(1 / r-\epsilon_{2}, 1 / r+\epsilon_{2}\right)$. Assume that $E\left(\gamma_{s_{0}}().\right)$ takes its maximum at the point $t_{0}$. Then

$$
0=\left.\frac{\mathrm{d}}{\mathrm{d} t} E\left(\gamma_{s_{0}}(t)\right)\right|_{t=t_{0}}=E^{\prime}\left(t_{0}\left(\bar{u}+s_{0} v\right) r\right)\left(\bar{u}+s_{0} v\right) r,
$$

and therefore $t_{0}=g\left(s_{0}\right)$. So by (45) one obtains that

$$
E\left(t_{0}\left(\bar{u}+s_{0} v\right) r\right)<E(\bar{u}) .
$$

Thus,

$$
E\left(t\left(\bar{u}+s_{0} v\right) r\right)<E(\bar{u}), \quad \forall t \in[0,1] .
$$

In other words, $\max _{t \in[0,1]} E\left(\gamma_{s_{0}}(t)\right)<E(\bar{u})$. This indeed shows that

$$
c \leq \max _{t \in[0,1]} E\left(\gamma_{s_{0}}(t)\right)<E(\bar{u})
$$

as desired. 
Acknowledgements We would like to thank the referee for carefully reading our manuscript and for giving us valuable comments that substantially improved the clarity of the original manuscript.

\section{References}

1. Aizicovici, S., Papageorgiou, N.S., Staicu, V.: Existence of multiple solutions with precise sign information for superlinear Neumann problems. Ann. Math. Pura Appl. (4) 188(4), 679-719 (2009)

2. Anello, G.: Existence of infinitely many weak solutions for a Neumann problem. Nonlinear Anal. 57(2), 199-209 (2004)

3. Ambrosetti, A., Brezis, J., Cerami, G.: Combined effects of concave and convex nonlinearities in some elliptic problems. J. Funct. Anal. 122(2), 519-543 (1994)

4. Bonanno, G., Candito, P.: Three solutions to a Neumann problem for elliptic equations involving the p-Laplacian. Arch. Math. (Basel) 80(4), 424-429 (2003)

5. Bonheure, D., Grumiau, C., Troestler, C.: Multiple radial positive solutions of semilinear elliptic problems with Neumann boundary conditions. Nonlinear Anal. 147, 236-273 (2016)

6. Bonheure, D., Grossi, M., Noris, B., Terracini, S.: Multi-layer radial solutions for a supercritical Neumann problem. J. Differ. Equ. 261, 455-504 (2016)

7. Bonheure, D., Noris, B., Weth, T.: Increasing radial solutions for Neumann problems without growth restrictions. Ann. Inst. H. Poincaré Anal. Non Linéaire 29(4), 573-588 (2012)

8. Bonheure, D., Serra, E.: Multiple positive radial solutions on annuli for nonlinear Neumann problems with large growth. NoDEA 18(2), 217-235 (2001)

9. Bonheure, D., Serra, E., Tilli, P.: Radial positive solutions of elliptic systems with Neumann boundary conditions. J. Funct. Anal. 265(3), 375-398 (2013)

10. Boscaggin, A., Colasuonno, A., Noris, B.: Multiple positive solutions for a class of p-Laplacian Neumann problems without growth conditions. In: ESAIM: COCV

11. Boscaggin, A., Colasuonno, F., Noris, B.: A priori bounds and multiplicity of positive solutions for p-Laplacian Neumann problems with sub-critical growth. In: Proceedings A of the Royal Society of Edinburgh

12. Clarke, F.: A classical variational principle for periodic Hamiltonian trajectories. Proc. Am. Math. Soc. 76, 186-188 (1979)

13. Colasuonno, F., Noris, B.: A p-Laplacian supercritical Neumann problem. Discrete Continuous Dyn. Syst. A 37(6), 3025-3057 (2017)

14. Cowan, C., Moameni, A.: Supercritical Neumann problems on non-radial domains. In: Transaction of AMS

15. Cowan, C., Moameni, A., Salimi, L.: Existance of solutions to supercritical Neumann problems via a new variational principle. Electron. J. Differ. Equ. 2017(213), 1-19 (2017)

16. Ekeland, I.: Periodic solutions of Hamiltonian equations and a theorem of P. Rabinowitz. J. Differ. Equ. 34, 523-534 (1979)

17. Ekeland, I.: Convexity Methods in Hamiltonian Mechanics. Springer, Berlin (1990)

18. Ekeland, I., Temam, R.: Convex Analysis and Variational Problems. American Elsevier Publishing Co. Inc., New York (1976)

19. Evans, L.C.: Partial Differential Equations. AMS, Providence (1998)

20. Faraci, F.: Multiplicity results for a Neumann problem involving the p-Laplacian. J. Math. Anal. Appl. 277(1), 180-189 (2003)

21. Filippakis, M., Gasiński, L., Papageorgiou, N.S.: Multiplicity results for nonlinear Neumann problems. Can. J. Math. 58(1), 64-92 (2006)

22. Gilbarg, D., Trudinger, N.S.: Elliptic partial differential equations of second order. Reprint of the 1998 edition. Classics in Mathematics. Springer, Berlin (2001)

23. Grossi, M., Noris, B.: Positive constrained minimizers for supercritical problems in the ball. Proc AMS 140(6), 2141-2154 (2012)

24. Kuhestani, N., Moameni, A.: Multiplicity results for elliptic problems with super-critical concave and convex nonlinearities. Calc. Var. Partial Differ. Equ. 57(2), 57 (2018)

25. Moameni, A.: Non-convex self-dual Lagrangians: new variational principles of symmetric boundary value problems. J. Funct. Anal. 260, 2674-2715 (2011)

26. Moameni, A.: A variational principle for problems in partial differential equations and analysis (Submitted)

27. Moameni, A.: A variational principle for problems with a hint of convexity. C. R. Math. Acad. Sci. Paris 355(12), 1236-1241 (2017) 
28. Serra, E., Tilli, P.: Monotonicity constraints and supercritical Neumann problems. Ann. Inst. H. Poincaré Anal. Non Linéaire 28(1), 63-74 (2011)

29. Secchi, S.: Increasing variational solutions for a nonlinear p-laplace equation without growth conditions. Ann. Mat. Pura Appl. 191(3), 469-485 (2012)

30. Szulkin, A.: Minimax principles for lower semicontinuous functions and applications to nonlinear boundary value problems. Ann. Inst. H. Poincaré Anal. Non Linéaire 3(2), 77-109 (1986) 